\title{
Importance and use of IOT in Healthcare
}

\author{
Abhinav Kumar \\ Student, MRIIRS \\ $257,2^{\text {nd }}$ Floor, Sector-21 \\ Rohini, Delhi-110086
}

\author{
Divyansh Dixit \\ Student, MRIIRS \\ DA Block 40C, Hari Nagar \\ New Delhi-110064
}

\author{
Akhilesh Kumar \\ Student, MRIIRS \\ 1534, Block-29, Sector-29 \\ Pushp Vihar, Saket
}

\author{
Amana Yadav \\ Assistant Professor \\ FET, MRIIRS
}

\begin{abstract}
The healthcare industry is in a condition of extraordinary sadness. Healthcare services are costlier than any time in recent memory.

What the world drawing nearer is where essential medicinal services would get far off to the vast majority, an enormous segment of society would go inefficient attributable to mature age and individuals would be more inclined to ceaseless illness. IoT application improvement is at the salvage. The utilization of the Internet of Things (IoT) in healthcare services (the business, individual medicinal services and social insurance installment applications) has forcefully expanded across different explicit Internet of Things use cases. Internet of Things has become an ultimate building block in the advancement of healthcare observing framework. The point of an effective IoT human services framework is to give ongoing distant observing of patient wellbeing condition, to forestall the critical patient conditions and to improve the quality of life through smart IoT environmental factors. The proposed framework here comprises of different clinical gadgets, for example, sensors and online or portable based applications which impart through system associated gadgets and assists with observing and record patients' wellbeing information and clinical data. The primary rationale is to manufacture a framework to give world-class clinical guide to the patients even in the remotest regions without any emergency clinics. The gathered data can be utilized to examine and anticipate interminable issues or different maladies, for example, coronary failures in starter stage itself utilizing the information mining methods that will likewise give the methodology favorable to dynamic.
\end{abstract}

\section{Keywords}

Internet of Things (IoT); Healthcare; sensors;

\section{INTRODUCTION}

The total populace is expanding immensely. Healthcare is a basic part of life. The serious issue that each patient, especially living in far off areas discovered was inaccessibility of specialists and treatment on basic conditions. This had repulsive results at the forefront of individuals' thoughts about the medical clinics and specialists' administrations. Clearly, an answer is required to diminish the weight on healthcare frameworks while proceeding to give top notch care to in danger patients. These days with the usage of new advances by utilizing IoT gadgets for social insurance checking framework, these issues have been arranged to gigantic degree. The Internet of Things offers a rising innovation to accomplish the following degree of wellbeing administrations. It guarantees for the reasonable, minimal effort, solid and convenient gadgets to be conveyed or inserted with the patients, so that to empower consistent systems administration between the patients, clinical gadgets and doctors.
The Internet of things is the between association of gadgets, applications, sensors and system network that upgrades these substances to accumulate and trade information. The distinctive quality of Internet of Things in the social insurance framework is the consistent observing a patient through checking different boundaries and furthermore construes a decent outcome from the historical backdrop of such steady observing. As there could be examples where the specialist couldn't be alarmed in time when there is a crisis, in spite of 24 hours of observing. This paper accordingly makes a one of a kind commitments in that it identifies every single key part of a start to finish Internet of Things human services framework, and proposes a nonexclusive model that could be applied to all IoT-based medicinal services frameworks.

It utilizes Remote Health Monitoring System constrained by Raspberry pi. Raspberry Pi is a little installment card-sized single-board microcontroller made to upgrade the fundamental software engineering training in universities and creating countries. A framework is intended to persistently screen the indispensable boundaries, for example, pulse, circulatory strain and internal heat level. The data is put away on a cloud worker database and can be shown through an online site or portable application by approved staff in particular. The previous is cultivated by utilizing MySQL $\mathrm{db}$. module to connect Raspberry pi to the database while the last is accomplished by the blend of Raspberry Pi and GSM module and the web interface. Concentrate is put on sensors for observing different wellbeing boundaries, short-and longextend correspondences guidelines, and cloud advances. The subsequent information is put away, prepared and analyzed with the current wellbeing records. Utilizing the accessible information records and choice emotionally supportive networks, the doctor can do a superior anticipation so that to propose early treatment. In any event, when the specialist isn't accessible, this investigation empowers the present machines to anticipate the medical problems. Not just forecast, machines can likewise have the option to come out with the prescriptions from the precise investigation of the therapeutic databases.

The utilization of the Internet of Things (IoT) in human services is an immense biological system. Inside the general associated social insurance and eHealth picture, more coordinated methodologies and advantages are looked for with a job for the alleged Internet of Healthcare Things (IoHT) or Internet of Medical Things (IoMT).

\section{IOT OVERVIEW}

IoT frameworks permit clients to accomplish further mechanization, examination, and reconciliation inside a framework. They improve the span of these zones and their exactness. IoT uses existing and developing innovation for detecting, systems administration, and mechanical technology.

IoT misuses late advances in programming, falling equipment costs, and current perspectives towards innovation. Its new 
and progressed components acquire significant changes the conveyance of items, merchandise, and benefits; and the social, financial, and political effect of those changes. The web of things (IoT) is a figuring idea that portrays a situation where regular physical items are associated with the web and can recognize themselves to different gadgets or cycles, by means of an IP address.[1]

The IoT is critical in light of the fact that an item that can speak to itself carefully becomes an option that could be more prominent than the article without anyone else. No longer does the article simply identify with the cycle; it presently interfaces with encompassing items and information base information, allowing "large information" investigation and experiences.

Specifically, "things" may discuss independently with different things and different gadgets, for example, sensors in assembling conditions or an action tracker with a cell phone. IoT has advanced from the assembly of remote advances, small scale electromechanical frameworks, microservices and the web. This combination has destroyed the dividers between operational innovation and data innovation, permitting unstructured machine-created information to be dissected for bits of knowledge that will drive upgrades. Shopper IoT took another progressive way, either by getting associated - for instance, speed sensors on a bicycle - or being recently created. In different cases, for example, in human services, things have been there yet not broadly utilized, for example, persistent wellbeing status.[2]

\section{WHY IOT FOR HEALTHCARE ?}

IoT empowers social insurance experts to be more attentive and associate with the patients proactively. Information gathered from IoT gadgets can assist doctors with recognizing the best treatment measure for patients and arrive at the normal results.

\subsection{Advantages}

\subsubsection{Simultaneous announcing and checking}

Continuous checking through associated gadgets can spare lives in occasion of a health related crisis like cardiovascular breakdown, diabetes, asthma assaults, and so forth. Advantages of iot wellbeing detailing and observing With constant checking of the condition set up by methods for a keen clinical gadget associated with a cell phone application, associated gadgets can gather clinical and other required wellbeing information and utilize the information association of the cell phone to move gathered data to a doctor. Focus of Connected Health Policy directed an investigation that demonstrates that there was a half decrease in 30-day readmission rate due to far off patient checking on cardiovascular breakdown patients. The IoT gadget gathers and moves wellbeing information: circulatory strain, oxygen and glucose levels, weight, and ECGs. These information are put away in the cloud and can be imparted to an approved individual, who could be a doctor, the insurance agency, a taking an interest wellbeing firm or an outside expert, to permit them to take a gander at the gathered information paying little mind to their place, time, or gadget.[3]

\subsubsection{End-to-end availability and moderateness}

IoT can robotize tolerant consideration work process with the assistance human services portability arrangement and other new advancements, and cutting edge social insurance offices. IoT in social insurance empowers interoperability, machineto-machine correspondence, data trade, and information development that makes human services administration conveyance viable. Availability conventions: Bluetooth LE,
Wi-Fi, Z-wave, ZigBee, and other current conventions, medicinal services faculty can change the manner in which they spot disease and afflictions in patients and can likewise advance progressive methods of treatment. Thusly, innovation driven arrangement cuts down the expense, by chopping down pointless visits, using better quality assets, and improving the designation and arranging. [4]

\subsubsection{Data variety and examination}

Immense measure of information that a human services gadget sends in an extremely brief timeframe inferable from their constant application is difficult to store and oversee if the admittance to cloud is inaccessible. In any event, for social insurance suppliers to procure information beginning from different gadgets and sources and break down it physically is an intense wagered. IoT gadgets can gather, report and examinations the information continuously and slice the need to store the crude information. This all can happen overcloud with the suppliers just gaining admittance to conclusive reports with charts. Also, social insurance tasks permit associations to get indispensable medicinal services investigation and information driven experiences which accelerate dynamic and is less inclined to mistakes. [5]

\subsubsection{Tracking and alarms}

On-time alert is basic in occasion of dangerous conditions. Clinical IoT gadgets assemble fundamental information and move that information to specialists for continuous following, while at the same time dropping warnings to individuals about basic parts through versatile applications and other connected gadgets. Reports and alarms offer a firm input about a patient's condition, independent of spot and time. It additionally enables settle on to knowledgeable choices and give on-time treatment. In this way, IoT empowers continuous alarming, following, and observing, which grants hands-on medicines, better exactness, able mediation by specialists and improve total patient consideration conveyance results. [6]

\subsubsection{Remote clinical help}

In occasion of a crisis, patients can contact a specialist who is numerous kilometers away with a savvy versatile applications. With versatility arrangements in medicinal services, the doctors can in a split second check the patients and distinguish the illnesses in a hurry. Likewise, various human services conveyance anchors that are guaging to fabricate machines that can disperse drugs based on patient's remedy and affliction related information accessible by means of connected gadgets. IoT will Improve the patient's consideration In clinic. This thus, will cut on individuals' spread on social insurance.[7]

\section{COMPONENTS NECESSARY FOR IT}

The different Components to be utilized in framework are :

\subsection{Raspberry Pi}

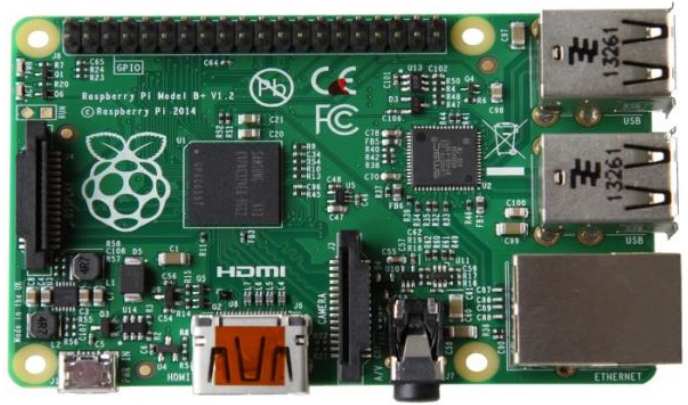

Fig4.1 
The Raspberry Pi is a bank card size microcontroller with the highlights of a little pc and is amazingly mainstream for advancement purposes since it offers the whole Linux worker and fringe gadget network on a solitary chip and is very practical. The GPIO pins accessible on the board are utilized for the information/yield reason and can be modified according to the need. For, the proposed framework there will be need of utilizing Raspberry Pi board rendition 3. The Raspberry $\mathrm{Pi}$ and IoT together end up being a pioneer advancement innovation in medicinal services framework.[8]

\subsection{Temperature Sensor}

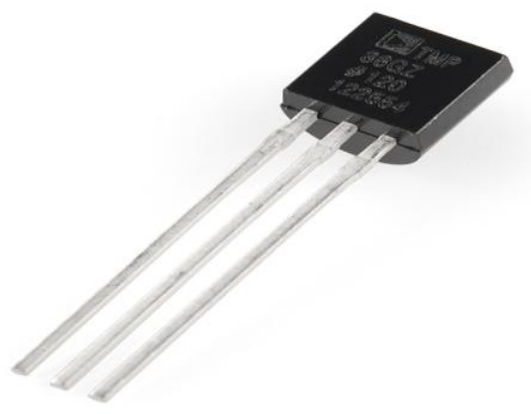

Fig4.2

For estimating the temperature LM35 sensor has been utilized which is an IC sensor used to quantify the temperature with the assistance of the simple yield corresponding to the temperature. The LM35 is an IC temperature sensor with a yield voltage which is corresponding to the Celsius temperature. [9] The LM35 is better than direct temperature sensors which have adjustment in Kelvin, since one doesn't have to eliminate a huge consistent voltage from the yield an incentive to acquire the Celsius perusing. These striking highlights of the LM35 sensor make interfacing to a circuit amazingly simple. [11]

\subsection{Heartbeat Sensor}

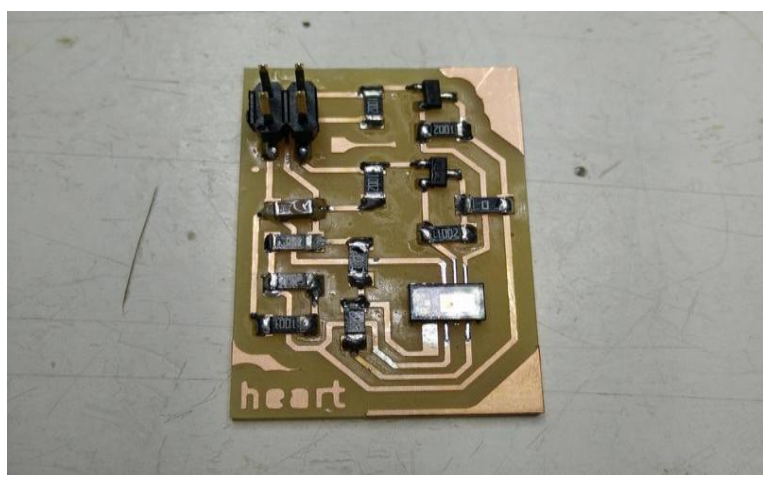

Fig4.3

The pulse is estimated utilizing a couple of LED and LDR and a microcontroller and it takes a shot at the essentials of optoelectronics. The infrared radiation is produced by IR drove and the infrared light is reflected by the surface. The power of radiation created electron-gap pair which thusly delivers spillage current. This current accordingly created is sent through a resistor to get the corresponding voltage. Hence, the more prominent is the force of the episode beam, the bigger estimation of voltage streaming across resistor will be acquired.[10] The pulse is estimated by setting the tip of pointer upon the sensor. When the circuit detects the beat, a
LED will begin flickering alongside the heartbeat. The yield is sent to a circuit or a smaller scale regulator to quantify the heart beat rate in BPM. [11]

\subsection{Vibration Sensor}

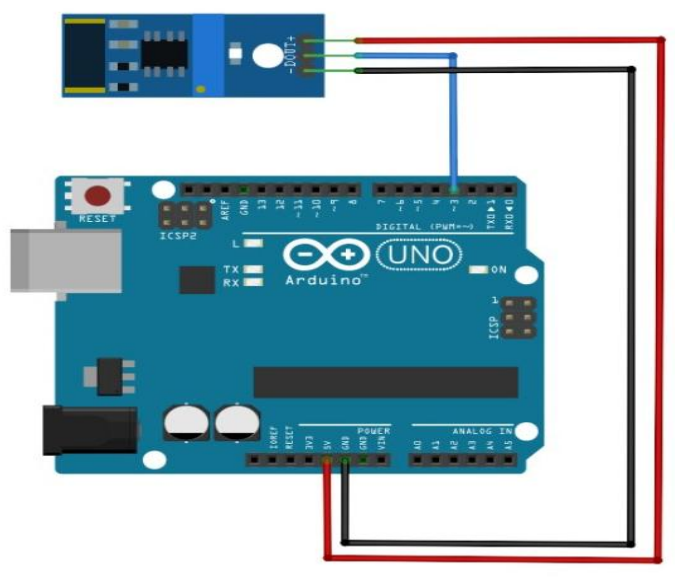

Fig4.4

The vibration sensor utilized in here faculties the shaking of the encompassing and subsequently device use it here to screen whether the patient is shuddering so legitimate guide can be given. Preferably, the two contacts of sensor don't contact one another. When by any outer power these two contacts contact one another and when the power is taken out the sensor terminals seperate. The on-board blue LED outwardly shows correspondence on the web and actuation.[11]

\subsection{BP Sensor}

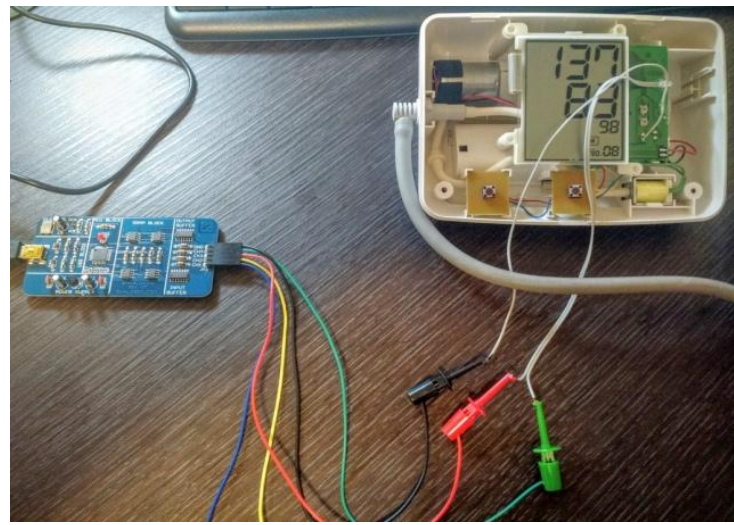

Fig.4.5

For estimating the pulse, there is a need to utilize here a manual circulatory strain screen rather than an advanced one as it is less expensive. It is usually known as a sphygmomanometer and the unit comprises of an arm sleeve, a crush bulb to expand the sleeve, stethoscope and a sensor to peruse the weight. Circulatory strain is measure utilizing a pneumatic force sensor. The readings are as electrical signs. These readings are additionally changed over to advanced structure to be perused by the Raspberry Pi.[11]

\subsection{ADC}




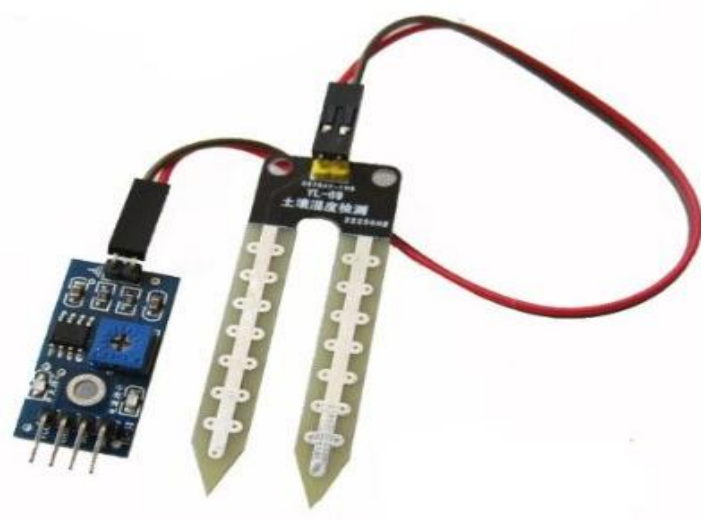

Fig.4.6

The MCP3008 is an ease 8-channel 10-piece simple to computerized converter. This chip is an extraordinary choice in the event that one needs to peruse straightforward simple signs, as from a temperature or light sensor. [11]

\subsection{GSM Module}

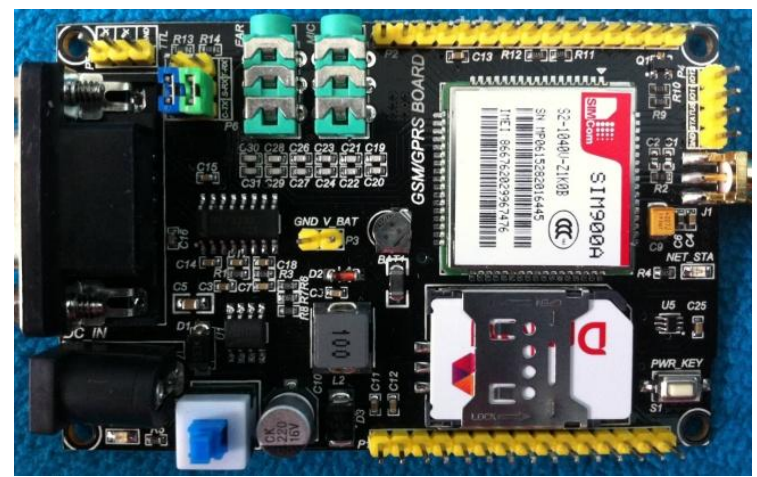

Fig.4.7

The GSM module utilized here in this paper is GPRS/GSM Quadband Module (SIM900) which offers GPRS association with this framework, and incorporates the SIM900 correspondence module from SIMCom. This module can acknowledge any sort of sim card having its own one of a kind number. The equivalent can be utilized to send messages, settle on decisions or make attachments to give web network.

The information from the above sensors is continually refreshed in MySQL data set which is connected to the web UI utilizing the python code. The patient can sign in and screen their wellbeing status whenever. The framework is made brilliant to trigger a SMS/Email alert through the best possible passages which guarantee a proficient conveyance of the message. Additionally, the qualities from these sensor in mix with different side effects which are asked from client dependent on starting finding is utilized to anticipate the malady persistent is experiencing, assuming any, utilizing the information mining procedure through this programming rationale and is shown because of examination alongside the subtleties of a specialist for the sickness in their general vicinity.[11]

\section{USE OF IOT IN HEALTHCARE}

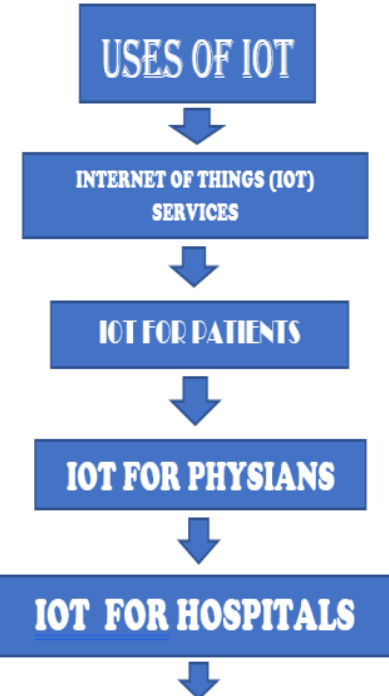

\section{IOT FOR HIDALTHINOURANGE}

Fig.5.1

Prior to Internet of Things, patients' associations with specialists were restricted to visits, and tele and text correspondences. There was no chance specialists or clinics could screen patients' wellbeing consistently and make suggestions as needs be.[12]

\subsection{Internet of Things (IoT) services}

It empowered gadgets have made far off checking in the medicinal services part conceivable, releasing the possibility to keep patients sheltered and solid, and enabling doctors to convey standout care. It has additionally expanded patient commitment and fulfillment as communications with specialists have become simpler and more effective. Moreover, distant observing of patient's wellbeing helps in decreasing the length of medical clinic remain and forestalls re-confirmations. IoT likewise majorly affects decreasing human services costs fundamentally and improving treatment results.[19]

IoT is without a doubt changing the human services industry by rethinking the space of gadgets and individuals communication in conveying social insurance arrangements. IoT has applications in human services that advantage patients, families, doctors, medical clinics and insurance agencies. [12]

\subsubsection{IoT for Patients}

Devices as wearables like wellness groups and other remotely associated gadgets like pulse and pulse checking sleeves, glucometer and so on give patients admittance to customized consideration. These gadgets can be tuned to remind carbohydrate level, practice check, arrangements, pulse varieties and considerably more. [13]

IoT has transformed people, particularly older patients, by empowering consistent following of wellbeing conditions. This majorly affects individuals living alone and their families. On any unsettling influence or changes in the normal exercises of an individual, ready component imparts signs to relatives and concerned wellbeing suppliers. 


\subsubsection{IoT for Physicians}

By utilizing wearables and other home checking hardware implanted with IoT, doctors can monitor patients' wellbeing all the more adequately. They can follow patients' adherence to therapy plans or any requirement for sure fire clinical consideration. IoT empowers medicinal services experts to be more attentive and associate with the patients proactively. Information gathered from IoT gadgets can assist doctors with recognizing the best treatment measure for patients and arrive at the normal results. [13]

\subsubsection{IoT for Hospitals}

Apart from checking patients' wellbeing, there are numerous different zones where IoT gadgets are extremely valuable in medical clinics. IoT gadgets labeled with sensors are utilized for following ongoing area of clinical hardware like wheelchairs, defibrillators, nebulizers, oxygen siphons and other observing gear. Sending of clinical staff at various areas can likewise be investigated continuous.

The spread of diseases is a significant worry for patients in medical clinics. IoT-empowered cleanliness observing gadgets help in keeping patients from getting contaminated. IoT gadgets additionally help in resource the board like drug store stock control, and ecological observing, for example, checking cooler temperature, and mugginess and temperature control. [13]

\subsubsection{IoT for Health Insurance Companies}

There are various open doors for wellbeing safety net providers with IoT-associated keen gadgets. Insurance agencies can use information caught through wellbeing observing gadgets for their guaranteeing and claims tasks. This information will empower them to recognize misrepresentation asserts and distinguish possibilities for guaranteeing. IoT gadgets acquire straightforwardness among guarantors and clients the guaranteeing, estimating, claims taking care of, and hazard evaluation measures. In the light of IoT-caught information driven choices in all activity measures, clients will have satisfactory perceivability into basic idea behind each choice made and cycle results.[14]

Safety net providers may offer motivations to their clients for utilizing and sharing wellbeing information created by IoT gadgets. They can remunerate clients for utilizing IoT gadgets to monitor their standard exercises and adherence to treatment plans and prudent wellbeing measures. This will assist safety net providers with lessening claims essentially. IoT gadgets can likewise empower insurance agencies to approve claims through the information caught by these gadgets.[14]

\section{APPLICATION}

This paper, has proposed a system in which the patient's interior warmth level, heartbeat, body improvements, and circulatory strain examining results that are being checked by the structure. The various sensors are put on the patient's body and they take the readings and give the relating sign to the raspberry pi.[18] The Raspberry $\mathrm{Pi}$ is a charge card estimated single-board PC that deals with Linux OS. Here, various sensors are used to measure the patient's inward warmth level, beat, Blood Pressure, and their specific results are sent to the database by methods for Raspberry Pi and can be seen from wherever worldwide through the web energized through the GSM module. The programming in Raspberry Pi is done in python language and it sends the data related to the patients' prosperity to the laborer related by methods for the Internet. The nuances can be adequately gotten online by genuine confirmation and the prosperity status of the patient can be checked.[15]

\subsection{Health Monitoring System}

This module involves the equipment parts of the framework that makes it IoT empowered and is utilized to record the wellbeing boundaries of the patient utilizing different sensors.[17] Here, Raspberry pi goes about as a focal worker to which all the sensors are associated through the GPIO pins or utilizing MCP3008 simple to-computerized convertor if their yield is in the simple structure as raspberry pi works just on advanced signs. The pi peruses the constant qualities and updates them to mySQL db which is then used to show them on the web interface.[13]

\subsection{Emergency Alert System}

This module in especially manages the means to be taken after a variation from the norm is distinguished in the soundness of patient, for example, telling his/her relative just as the medical clinic. IOT have set up certain edge esteems in the program which whenever crossed will trigger a caution as email/SMS to the patients family/specialist. [15]

\subsection{Health Status Prediction System}

This is one of the most encouraging modules of the framework. In this module, device utilize the patients' wellbeing information as recorded by the framework alongside any side effects they might be feeling by posing a couple of straightforward inquiries and contrast it and the current information base to anticipate if any ailment/issue the patient may have consequently making it a proficient Expert System with legitimate information mining methods.[15]

\section{CHALLENGES FOR IOT IN HEALTHCARE?}

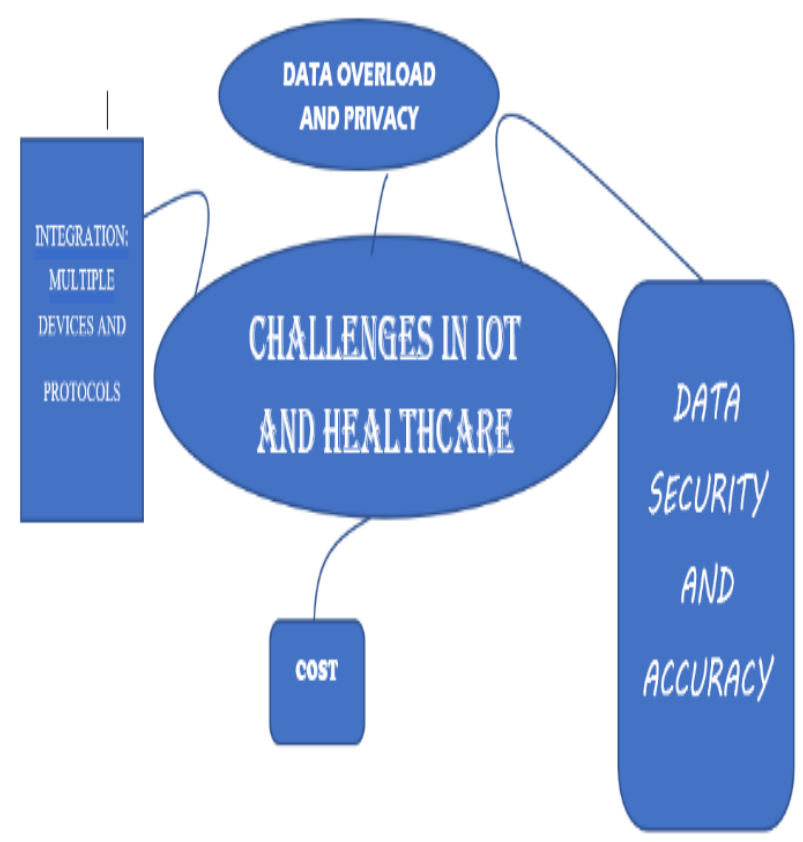

Fig.7.1

\subsection{Data security \& privacy}

One of the most noteworthy dangers that IoT presents is of information security and protection. IoT gadgets catch and send information continuously. In any case, the majority of the IoT gadgets need information conventions and norms. Notwithstanding that, there is huge uncertainty with respect to 
information proprietorship guideline. Every one of these elements make the information profoundly defenseless to cybercriminals who can hack into the framework and bargain Personal Health Information (PHI) of the two patients just as specialists. Cybercriminals can abuse patient's information to make counterfeit IDs to purchase medications and clinical gear which they can sell later. Programmers can likewise record a false Insurance guarantee in patient's name.

\subsection{Integration: multiple devices \& protocols}

Mix of different gadgets likewise causes deterrent in the execution of IoT in the human services division. The explanation behind this obstruction is that gadget makers haven't arrived at an agreement with respect to correspondence conventions and standard. Along these lines, regardless of whether the assortment of gadgets are associated; the distinction in their correspondence convention entangles and blocks the cycle of information total. This nonconsistency of the associated gadget's conventions hinders the entire cycle and decreases the extent of adaptability of IoT in medicinal services.

\subsection{Data overload \& accuracy}

As examined before, information total is troublesome because of the utilization of various correspondence conventions and principles. Be that as it may, IoT gadgets despite everything record a huge amount of information. The information gathered by IoT gadgets are used to increase imperative bits of knowledge. In any case, the measure of information is colossal to such an extent that getting experiences from it are getting incredibly hard for specialists which, at last influences the nature of dynamic. Additionally, this worry is ascending as more gadgets are associated which record increasingly more information.

\subsection{Cost}

Shocked to see cost contemplations in the test segments? I know the majority of people would be; however the primary concern is: IoT has not made the medicinal services encourages reasonable to the average person yet. The blast in the Healthcare costs is a stressing sign for everyone particularly the created nations. The circumstance is with the end goal that it offered ascend to "Clinical Tourism" in which patients with basic conditions access human services offices of the creating countries which costs them as less as onetenth. IoT in medicinal services as an idea is an intriguing and promising thought.

Notwithstanding, it hasn't illuminated the cost contemplations starting at now. To effectively execute IoT application advancement and to pick up its complete enhancement the partners must make it savvy else it will consistently stay out of everybody's scope with the exception of the individuals from the high class.

\section{RESULT}

The patient can check their wellbeing status whenever from the solace of their homes and visit medical clinics just when they truly need to. This should be possible by utilizing this framework whose outcome is brought on the web and can be seen from anyplace around the globe. Since it is a model, this framework shows the practically continuous estimations of different wellbeing boundaries and copies of how the equivalent can be actualized in reality. The specialists can likewise utilize the $\log$ of the patient body condition to consider and decide the impact of medication or other such things. The keen expectation module predicts the illness that the patient is experiencing by approaching them for different manifestations they may have and the alternatives depend on the past side effect. The last end is made after at any rate 3-4 indications are distinguished.

\section{CONCLUSION}

IoT changes how the offices are conveyed to the medicinal services industry. These innovations improve the item, causing a bigger impact by uniting minor changes. The framework can be additionally improved further by adding man-made reasoning framework segments to encourage the specialists and the patients. The information, comprising clinical history of numeroour patients' boundaries and comparing results, can be investigated utilizing information mining, looking for predictable examples and methodical connections in the illness. For example, if a patient's wellbeing boundaries are changing in a similar example as those of a past patient in the information base, the outcomes can likewise be assessed. If the comparative examples are found over and again, it would be simpler for the specialists and clinical scientists to discover a solution for the issue. With the above issues distinguished a framework despite everything should be structured where the doctor at other area can examine the continuous patient's essential boundaries through secure systems. A framework should be structured in order to give a total protection from various assaults, with access control and validation convention fused for IoT based healthcare services.

\section{REFERENCES}

[1] P.V. Dudhe ; N.V. Kadam ; R. M. Hushangabade ; M. S. Deshmukh Internet of Things (IOT): An overview and its applications .2017 International Conference on Energy, Communication, Data Analytics and Soft Computing (ICECDS).

[2] Antar Shaddad Abdul-Qawy, Pramod P. J, E. Magesh, T. Srinivasulu. The Internet of Things (IoT): An Overview A S Abdul-Qawy et al. Int. Journal of Engineering Research and Applications .ISSN: 2248-9622, Vol. 5, Issue 12, (Part - 2) December 2015, pp.71-82

[3] Stephanie B. Baker ; Wei Xiang ; Ian Atkinson Internet of Things for Smart Healthcare: Technologies, Challenges, and Opportunities IEEE Access (Volume: 5 ).

[4] Gulraiz J. Joyia, Rao M. Liaqat, Aftab Farooq, and Saad Rehman. Internet of Medical Things (IOMT): Applications, Benefits and Future Challenges in Healthcare Domain. Journal of Communications Vol. 12, No. 4, April 2017.

[5] S. M. R. islam, D. Kwak, M. H. Kabir, M. Hossain, and K. Kwak, "The internet of things for HealthCare: A comprehensive survey," IEEE Access, 2015.

[6] W.-Y. Chung, Y.D.L., Jung, S.J.: A cooperative Internet of Things (IoT) for rural healthcare monitoring and control. In: A wireless sensor network compatible wearable u-healthcare monitoring system using integrated ECG, accelerometer and SpO2. pp. 1529-1532 (2008)

[7] Y. J. Fan, Y. H. Yin, L.D.X.Y.Z., Wu, F.: IoT-based smart rehabilitation system. In: IEEE Trans. Ind. Informat. pp. 1568-1577 (2014) 
[8] M. Richardson and S. Wallace, Getting started with Raspberry Pi, O’Reilly, USA, 2013

[9] T. Kuglestadt, Semiconductor Temperature Sensors Challenge Precision RTDs and Thermistors in Building Automation, Application Report, SNAA267-April 2015,

[10] C. K. Das, M. W. Alam and M. I. Hoque, "A Wireless Heartbeat And Temperature Monitoring System For Remote Patients", ICMERE2013-PI-197

[11] Shubham Banka, Isha Madan and S.S. Saranya Smart Healthcare Monitoring using IoT. International Journal of Applied Engineering Research ISSN 0973-4562 Volume 13, Number 15 (2018) pp. 11984-11989

[12] Himadri Nath Saha, Supratim Auddy, Subrata Pal: Health Monitoring using Internet of Things (IoT), IEEE Journal pp.69-73, 2017

[13] P. Verma and S. K. Sood, "Fog assisted-IoT enabled patient health monitoring in smart homes," IEEE Internet of Things Journal, vol. 5, no. 3, pp. 1789-1796, 2018.View at: Publisher Site | Google Scholar.
[14] Sullivan, H.T., Sahasrabudhe, S.: Envisioning inclusive futures: technology-based assistive sensory and action substitution. Futur. J. 87, 140-148 (2017)

[15] R. Shah and A. Chircu, "IOT and AI IN healthcare: a systematic literature review," Issues in Information Systems, vol. 19, 2018.

[16] Amine Rghioui, Abdelmajid Oumnad. Challenges and Opportunities of Internet of Things in Healthcare International Journal of Electrical and Computer Engineering (IJECE) Vol. 8, No. 5, October 2018, pp. 2753 2761.

[17] Mrunal Anekar, Aditi Khetarpal and S S Sambare. Survey on Real-Time Health Monitoring System based on IOT. International Journal of Computer Applications 181(28):19-23, November 2018.

[18] Fayez Hussain Alqahtani. The Application of the Internet of Things in Healthcare. International Journal of Computer Applications 180(18):19-23, February 2018.

[19] Pooja M. and Deepthi Das. Comparative Analysis of IoT based Healthcare Architectures. International Journal of Computer Applications 161(10):33-37, March 2017. 